\title{
CRÂNIOS PERUANOS EVIDENCIAM PADRÕES DE ATIVIDADE DIFERENTES ENTRE HOMENS E MULHERES
}

Há mais de 100 anos um conde inglês encontrou dois antigos cemitérios próximos ao mar, localizados em Pasamayo, a 30 milhas ao norte de Callao, Peru. Algumas das covas (individuais e múltiplas) já haviam sido abertas, tanto que o solo do local estava coberto de ossos. $O$ conde frisa ainda que algumas das mandíbulas apresentavam coloração esverdeada devido às moedas que lhes eram colocadas na boca. Provavelmente o cemitério havia sido escavado pelos conquistadores espanhóis, representando, portanto, nativos proto-históricos (Busk 1873). Como não se trata de uma escavação realizada profissionalmente, não se dispõe de mais dados arqueológicos. Entretanto, sabe-se que várias das populações costeiras antigas do Peru baseavam sua subsistência em pesca, coleta de vegetais e agricultura de irrigação (Fagan 1995).

Os 36 crânios da coleção de Pasamayo alocada no Museu de História Natural de Londres, foram estudados no intuito de verificar a existência e expressão de traços patológicos que pudessem assinalar padrões de atividade diferentes entre homens e mulheres e, portanto, a divisão de trabalho e/ou hierarquização da sociedade. Do total (Gráfico 1-A), 16 espécimes são do sexo masculino, 12 do feminino e 8 indeterminados. A impossibilidade de se atribuir o sexo a todos os indivíduos de uma coleção é esperada, pois os caracteres sexuais secundários (expressos pela robustez no sexo masculino) e as diferenças funcionais (relacionadas à passagem do feto através da pelvis no sexo feminino) somente se completam no final da adolescência (Saunders 1992). Desta maneira, a atribuição de sexo é impossível em juvenis, assim como também o é em alguns indivíduos adultos. Por isso, os casos indeterminados foram excluídos da presente análise.

Escolhemos três traços que teoricamente poderiam apresentar freqüência diferente entre os sexos: lesões cranianas (Figura 1) como possível indicador de conflitos interpessoais, exostoses do meato auditivo (Figura 2) relacionadas com ativida- des de subsistência, e cribra orbitália e hiperostose porótica (Figuras 3,4 ) ligadas, por sua vez, à diferenças quanto a dieta ou resposta a doenças.

Como mostra o Gráfico 1-B, lesões cranianas são significativamente mais freqüentes $(p<0.05)$ em homens $(56 \%)$ do que em mulheres $(8 \%)$. Como se sabe, os povos andinos eram guerreiros e levando-se em consideração a contemporaneidade dos indivíduos aqui estudados com os conquistadores hispânicos, esta alta freqüência sugere, a princípio, um elevado grau de conflitos interpessoais. Entretanto, traumas cranianos podem também decorrer de quedas ou mesmo trepanações, ou seja, operações que consistem na remoção cirúrgica de porções de calota craniana, na tentativa de aliviar dores, doenças mentais ou até como ritual religioso (Ortner 1981). Apesar de a distribuição geográfica deste tipo de operação praticada desde o Neolítico (Alt et al 1997) até o século XX (Oakley et al 1959) ser extensa e incluir Europa, ilhas do Pacífico, África do Norte e Oriente Médio, a trepanação é mais freqüente na América do Sul de onde provêm mais de 1000 dos casos descritos (Stewart 1958b). Outras causas possíveis de lesões cranianas incluem infecções, neoplasias, ou ação de fungos (Mann \& Murphy 1990). Como não dispusemos de microscópio por ocasião do estudo do material, não foi possível realizar o diagnóstico diferencial. Entretanto, como $90 \%$ das lesões observadas nos crânios de Pasamayo encontram-se cicatrizadas ou em vias de cicatrização, podemos afirmar que não foram diretamente fatais, quaisquer que tenham sido suas causas, a não ser que tenham originado doenças sistêmicas que levariam à morte mais tarde.

A exostose do meato auditivo também resultou ser mais freqüente ( $\mathrm{p}=0.08)$ dentre os homens (39\%) que dentre as mulheres (8\%) de Pasamayo (Gráfico 1-C). Trata-se de um tumor benigno, observado desde o Neolítico (Arriaza et al 1993) até os dias de hoje, cujo crescimento tem sido atribuído a fatores genéticos (Berry \& Berry, 1967; Rothham- 
Notas - Rev. do Museu de Arqueologia e Etnologia, São Paulo, 8: 313-318, 1998.

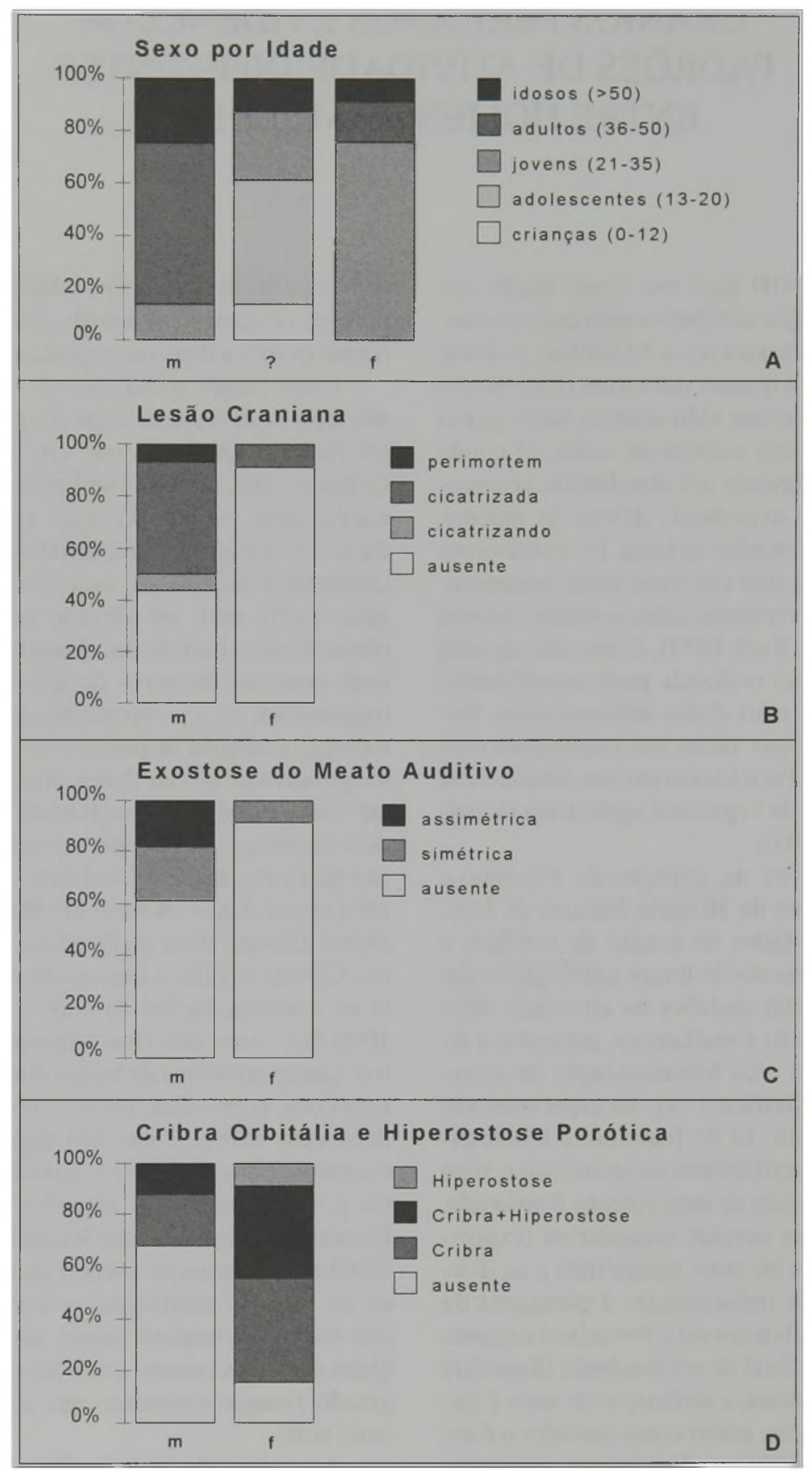

Gráfico 1 - Distribuição de freqüência de sexo por idade (A), lesão craniana por sexo $(B)$, exostose do meato auditivo por sexo $(C)$, cribra orbitália e hiperostose porótica por sexo $(D)$ na coleção de crâhiơs protohistóricos de Pasamayo, Peru. 
Notas - Rev. do Museu de Arqueologia e Etnologia, São Paulo, 8: 313-318, 1998.

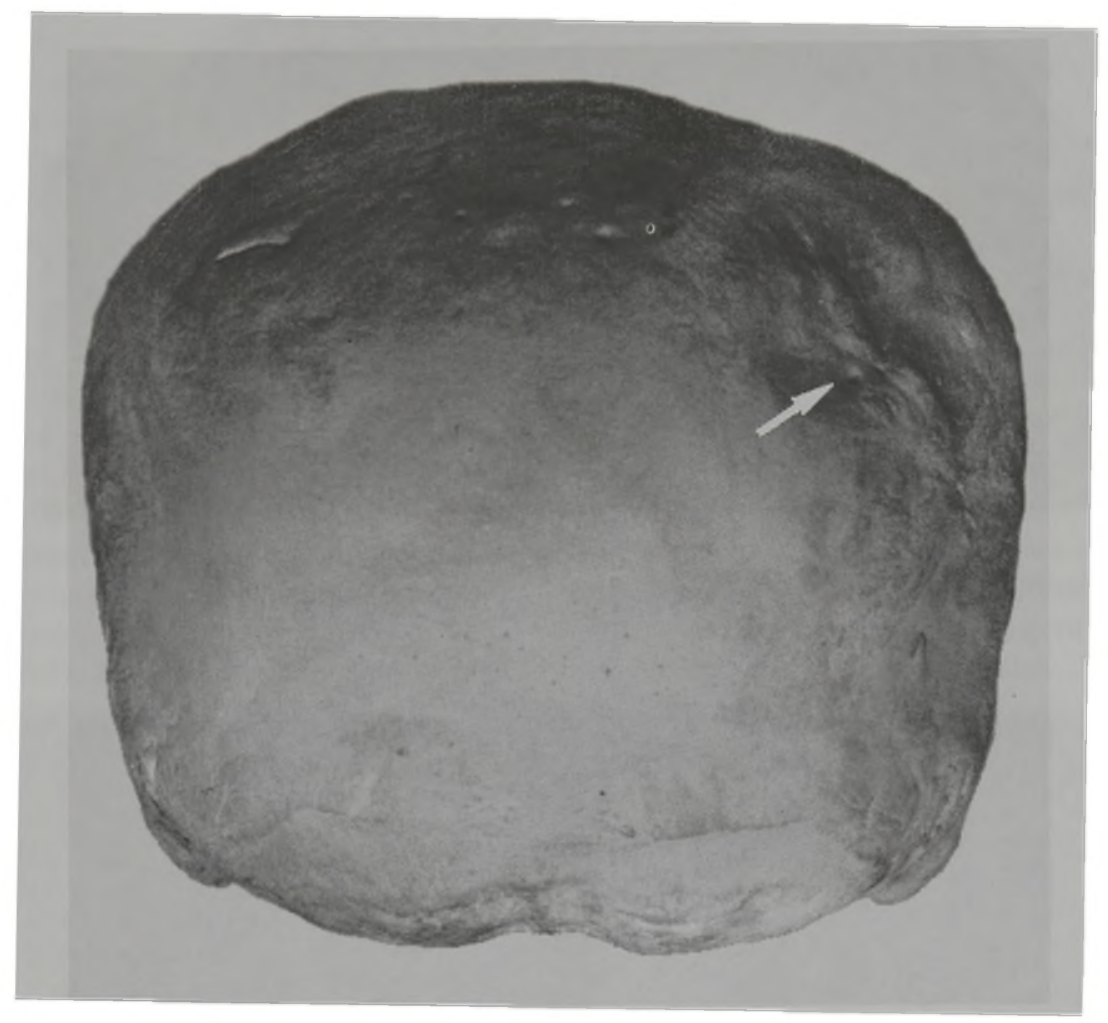

Figura 1 - Lesão craniana.

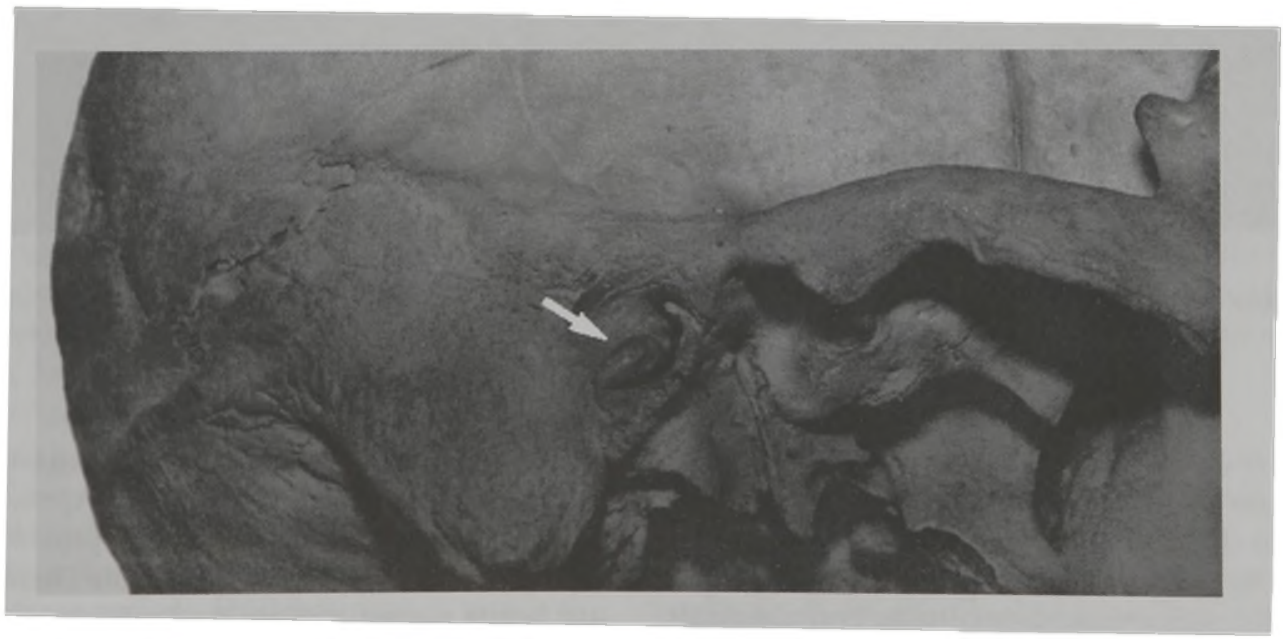

Figura 2 - Exostose do meato auditivo. 


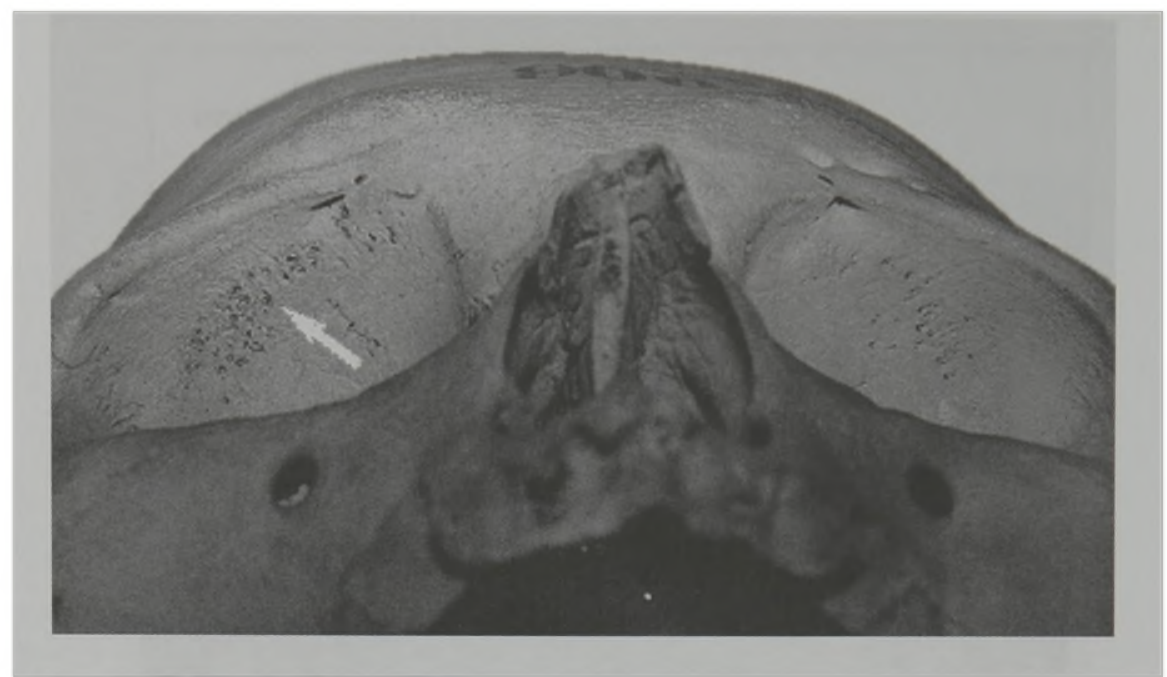

Figura 3 - Cribra orbitália.

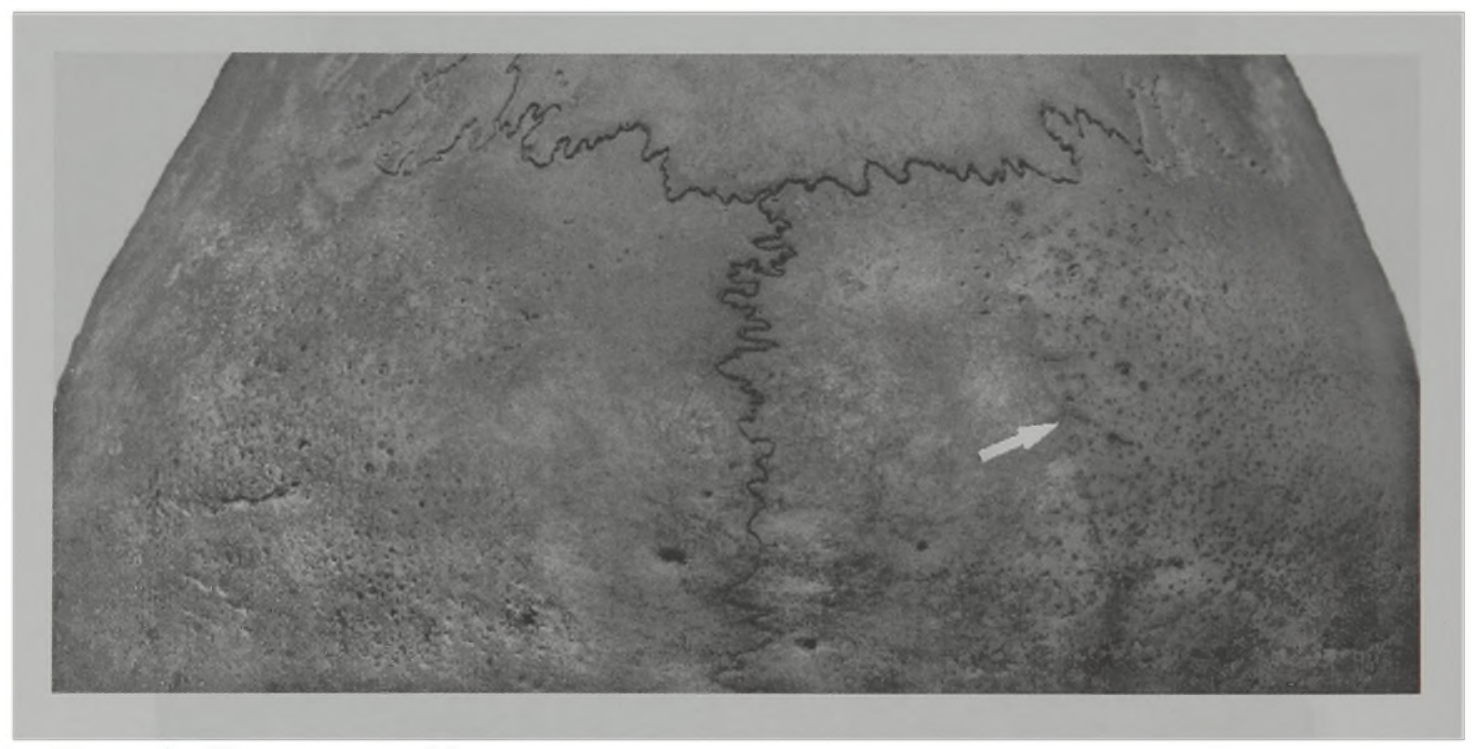

Figura 4-Hiperostose porótica.

mer et al 1984) ou ambientais, como a exposição a águas muito frias durante atividades como o mergulho (Dibartolomeo 1979, Filipo et al 1982). A hipótese ambiental parece mais plausível, uma vez que foi clinicamente demonstrada. Principalmente populações costeiras (Standen et al 1985) e aquelas que habitam regiões a $30-45^{0}$ de latitude com temperatura média das águas inferior a $19^{\circ} \mathrm{C}$ (Kennedy, 1986) apresentam maior incidência deste traço que, além disso, é sempre mais pronunciado em homens do que mulheres (Standen et al 1997). Apesar de a população aqui estudada ter vivido próximo ao equador (a $12^{0}$ de latitude), a corrente Humboldt que banha a costa pacífica da América do Sul é fria, reforçando, portanto, também no Peru, a hipótese da incidência de a exostose estar relacionada com fatores ambientais e não genéticos e de ser um traço típico de homens de regiões costeiras. 
Como se espera de uma antiga população peruana costeira (Hrdlicka 1914), a frequiência de cribra orbitália atinge $50 \%$ e a de hiperostose porótica 25\% dos crânios de Pasamayo. Apesar de descritas em trabalhos antigos, a etiologia de ambas ainda está sendo discutida. A cribra pode ser decorrente de anemia ferro-priva, malnutrição, escorbuto, hemorragia gastrointestinal, ancilostomíase, doenças endêmicas (Hirata 1988), ou por dieta baseada em alimentos que reduzem a absorção de ferro pelo organismo (Cohen 1984). A hiperostose, por sua vez, pode ser conseqüência de anemia ferro-priva hereditária ou nutricional, decorrente de diarréia de origem parasitária, deficiência de vitaminas, agentes tóxicos ou infecciosos (Mann \& Murphy 1990). Ambas as patologias podem ser encontradas em associação, e pelas suas características morfológicas e demográficas parecem estar fisiologicamente relacionadas (StuartMacadam 1989). Portanto, considerando-se uma e/ou outra, resulta que sua frequiência é significativamente maior $(\mathrm{P}<0,01)$ nas mulheres $(83 \%)$ do que nos homens (31\%) de Pasamayo (Gráfico 1D). Não dispomos dos esqueletos pós-cranianos desta coleção para verificarmos a existência de outras patologias que pudessem explicar a alta freqüência de cribra e hiperostose. Apesar de não termos certeza sobre o horizonte cronológico em que a população de Pasamayo vivia, é interessante notar que a partir de 5500 a.C., desenvolveu-se a agricultura em localidades próximas, possivelmente estimulada pelo fenômeno do El Niño que já assolava a humanidade em tempos remotos (Raymond 1981). Dentre as espécies cultivadas, o milho era uma das principais, caracterizando-se pelo efeito conhecido da inibição da absorção de ferro pelo organismo (Cohen 1984). A alta frequiência de cribra e hiperostose em mulheres de Pasamayo, portanto, poderia ser um reflexo da dieta diferencial entre os sexos. Esta é uma das hipóteses. Entretanto, há outros fatores que poderiam ter contribuído para tal.

A distribuição da idade de óbito (Gráfico 1A) evidencia que significativamente mais $(\mathrm{P}<0.01)$ mulheres $(75 \%)$ do que homens $(13 \%)$ faleciam entre 21 e 35 anos de idade, o que corresponde ao período fértil feminino. É provável que certa parte das mulheres tenha falecido durante ou logo após o parto (como é fato em tantas outras populações antigas e contemporâneas). Entretanto, não há evidência material nem informação arqueológica sobre bebês ou recém-nascidos nesta coleção.

Apesar de não podermos afirmar com certeza as causas que levaram aos indicadores osteológicos observados, e as inferências, portanto, serem especulativas, os dados desta coleção mostram claramente que há grandes diferenças entre os sexos. Os homens de Pasamayo, apesar de sofrerem mais lesões cranianas gozavam de uma expectativa de vida mais longa. A maior frequiência de exostoses mostra que estes homens eram sujeitos a fatores ambientais diferentes comparados às mulheres, pela sua atribuição de coletar alimentos submarinhos nas águas geladas do Pacífico. Por outro lado, a maior incidência de cribra orbitália e hiperostose porótica e a expectativa de vida menor em mulheres do que homens parecem confirmar o tratamento diferente que elas recebiam. Esta condição estaria possivelmente relacionada ao stress alimentar, que levaria a baixas respostas imunológicas e, por conseqüência, ao aumento de infecções oportunistas. Este quadro seria ainda agravado pela gestação e longos períodos de amamentação, levando algumas mulheres à morte em pleno período de reprodução.

Entretanto, apesar de raras, lesões cranianas e exostoses também foram observadas em mulheres, e cribra e hiperostose em homens, o que indica que as atividades que levaram à formação destes indicadores osteológicos não eram exclusivas de um ou outro sexo.

Este é um estudo preliminar. Análises mais profundas de outras patologias (como as orais ou aquelas relacionadas com artroses) podem futuramente esclarecer algumas das questões levantadas. De qualquer maneira, fica claro que o estudo de osteologia humana pode nos fornecer um quadro vivo e detalhado das atividades exercidas por sociedades antigas.

\section{Agradecimentos}

Este estudo teria sido inviável sem o apoio do curador Dr. R. Kruzsynski do Museu de História Natural de Londres. Apoio financeiro foi concedido pela FAPESP. 


\section{Referências bibliográficas}

ALT, K.; JEUNESSE, C.; BUITRAGO-TÉLLES, C.H.; WÄCHTER, R.; BOËS, E.; PICHLER, S.L.

1997 Evidence for Stone Age Cranial Surgery. $\mathrm{Na}$ ture, 387: 360.

ARRIAZA, B.; AUFDERHEIDE, A.; MUNOZ, I.

1993 Análisis antropológico físico de la inhumación de Acha 2. I. Munoz, B. Arriaza, A. Aufderheide (Eds.) Acha 2 y los orígines del poblamiento humano en Arica. Arica, Universidad de Tarapacá: 47-64.

BERRY, A.; BERRY, R.

1967 Epigenetic Variation in the Human Cranium. J Anat, 101: 361-379.

BUSK, F.R.S.

1873 Remarks on a Collection of 150 Ancient Peruvian Skulls. J Anthropol Inst, ii: 86-94. Dibartolomeo J. (1979) Exostoses of the External Auditory Canal. Ann Otol Rhinol Laryngol, 88 (suppl): 2-22.

COHEN, M.N.

1984 Health and Disease in the Rise of Civilization. New Haven, Londres, Yale University Press.

FAGAN, B.

1995 People of the Earth: An Introduction to World Prehistory (8th edition). New York, Harper Collins College Publishers.

FILIPO, R.; FABIANI, M.; BARBARA, M

1982 External ear canal exostosis: a physiopathological lesion in aquatic sports. $J$ Sports Med Phys Fitness, 22: 329-339.

GLEN-HADUCH, E.; SZOSTEK, K.; GLAB, H.

1997 Cribra orbitalia and trace element content in human teeth from neolithic and early bronze age graves in southern Poland. Am J Phys Anthropol, 103: 201-207.

HIRATA, K.

1988 A contribution to the paleopathology of cribra orbitalia in the Japanese. 1 Cribra orbitalia in Edo Japanese. St Marrianna Med J, 16: 6.

HRDLICKA, A

1914 Anthropological works in Peru in 1913 with notes onthe pathology of the ancient Peruvians. Washington, Smithsonian Institution.

\section{KENNEDY, G.E}

1986 The relationship between auditory exostoses and cold water: A latitudinal analysis. Am J Phys Anthropol, 71: 401-415.
MANN, R.W.; MURPHY, S.P.

1990 Regional Atlas of Bone Disease: a Guide to Pathologic and Normal Variation in the Human Skeleton. Springfield, Illinois, C Thomas.

OAKLEY, K.P.; BROOKE, M.A.; AKESTER, A.R.; BROTHWELL, D.R.

1959 Contributions on Trepanning or Trepanation in Ancient and Modern Times. Man, 59: 92-96.

ORTNER, D.J.; PUTSCHAR, W.G.J.

1981 Identification of pathological conditions in human skeletal remains. Smithsonian contributions to Anthropology, number 28. Washington DC: Smithsonian Institution Press.

RAYMOND, J.S.

1981 The maritime foundations of andean civilization: a reconstruction of the evidence. Am Antiquity, 46: 806-820.

ROTHHAMMER, F.; COCILOVO, J.; QUEVEDO, S

1984 Microevolution in Prehistoric Andean Populations: Chronologic non-metrical Variation in Northern Chile. Am J Phys Anthropol, 65: 157 162.

SAUNDERS, $S$.

1992 Subadult Skeletons and Growth related Studies. In Skeletal Biology of Past Peoples: Research Methods. SR Saunders \& MA Katzenberg (Eds.), New York: Wiley-Liss.

STANDEN, V.; ALLISON, M.; ARRIAZA, B.

1985 Osteoma del conducto auditivo externo: hipótesis en torno a una posible patología laboral prehispánica. Chungara, 15: 197-209.

STANDEN, V.G.; ARRIAZA, B.T.; SANTORO, C.M.

1997 Exernal auditory exostosis in prehistotic Chilean Populations: a test of the cold water hypothesis. Am J Phys Anthropol, 103: 119-129.

STEWART, T.D.

1958b Stone Age Skull Surgery: A General Review with Emphasis on the New World. Ann Rep Smithsonian Inst, 1957: 469-491.

STUART-MACADAM, P.L.

1989 Porotic hiperostosis: representative of childhood condition. Am J Phys Anthropol, 66: 391-398.

Sabine Eggers* Marta Mirazón Lahr* 